\title{
Improvement of cis,cis-Muconic Acid Production in Saccharomyces cerevisiae through Biosensor-Aided Genome Engineering
}

Guokun Wang, Süleyman Øzmerih, Rogério Guerreiro, Ana C. Meireles, Ana Carolas, Nicholas Milne, Michael K. Jensen, Bruno S. Ferreira, and Irina Borodina*

Cite This: ACS Synth. Biol. 2020, 9, 634-646

Read Online

ABSTRACT: Muconic acid is a potential platform chemical for the production of nylon, polyurethanes, and terephthalic acid. It is also an attractive functional copolymer in plastics due to its two double bonds. At this time, no economically viable process for the production of muconic acid exists. To harness novel genetic targets for improved production of cis,cis-muconic acid (CCM) in the yeast Saccharomyces cerevisiae, we employed a CCM-biosensor coupled to GFP expression with a broad dynamic response to

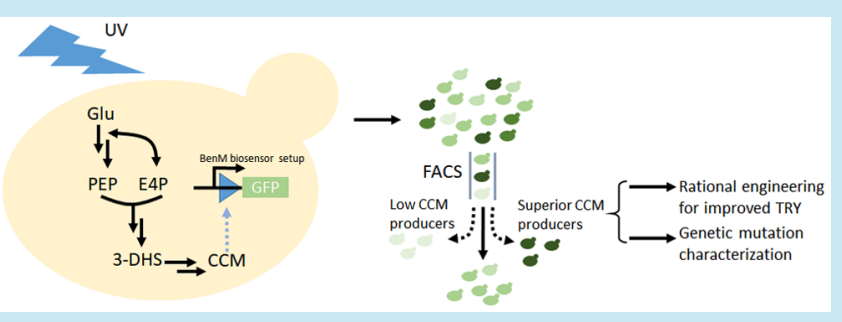
screen UV-mutagenesis libraries of CCM-producing yeast. Via fluorescence activated cell sorting we identified a clone Mut 131 with a $49.7 \%$ higher CCM titer and 164\% higher titer of biosynthetic intermediate-protocatechuic acid (PCA). Genome resequencing of the Mut131 and reverse engineering identified seven causal missense mutations of the native genes (PWP2, EST2, ATG1, DIT1, CDC15, CTS2, and MNE1) and a duplication of two CCM biosynthetic genes, encoding dehydroshikimate dehydratase and catechol 1,2-dioxygenase, which were not recognized as flux controlling before. The Mut131 strain was further rationally engineered by overexpression of the genes encoding for PCA decarboxylase and AROM protein without shikimate dehydrogenase domain (Aro $1 \mathrm{p}^{\Delta \mathrm{E}}$ ), and by restoring URA3 prototrophy. The resulting engineered strain produced $20.8 \mathrm{~g} / \mathrm{L} \mathrm{CCM}$ in controlled fed-batch fermentation, with a yield of $66.2 \mathrm{mg} / \mathrm{g}$ glucose and a productivity of $139 \mathrm{mg} / \mathrm{L} / \mathrm{h}$, representing the highest reported performance metrics in a yeast for de novo CCM production to date and the highest production of an aromatic compound in yeast. The study illustrates the benefit of biosensor-based selection and brings closer the prospect of biobased muconic acid.

KEYWORDS: biosensor, muconic acid, mutagenesis, Saccharomyces cerevisiae, reverse engineering

$\mathrm{M}$ uconic acid is a six-carbon dicarboxylic acid with two conjugated double bonds. Depending on the geometry of the double bonds, muconic acid exists in three conformations, cis,cis-muconic acid (CCM), trans,trans-muconic acid, and cis,trans-muconic acid. Muconic acid has various potential applications in the manufacture of new functional resins, plastics, nylon, agrochemicals, and pharmaceuticals. ${ }^{1,2}$ Currently, muconic acid is not in supply as a commodity chemical in a commercially available manner because there are no economically viable petrochemical or microbial manufacturing processes for its production.

A few microbes can produce muconic acid naturally as a degradation product of some aromatic compounds; for example, Pseudomonas putida can produce CCM from catechol $^{3}$ via catechol 1,2-dioxygenase. When the gene encoding catechol 1,2-dioxygenase was overexpressed in $P$. putida KT2440, the strain could produce $64.2 \mathrm{~g} / \mathrm{L} \mathrm{CCM}$ from catechol. ${ }^{4}$ The substrate spectrum was extended to the lignin monomer, phenol, and $p$-coumaric acid, by implementation of heterologous enzymes, phenol hydroxylase, and protocatechuic acid (PCA) decarboxylase (AroY), respectively. The resulting strains thereby produced $13 \mathrm{~g} / \mathrm{L}$ CCM on hydrothermally treated softwood lignin ${ }^{4}$ or $13.5 \mathrm{~g} / \mathrm{L} \mathrm{CCM}$ from glucose and $p$ coumaric acid. $^{5}$

The common microbial platforms Escherichia coli and Saccharomyces cerevisiae have also been engineered to produce CCM. Recombinant E. coli cells expressing catechol 1,2dioxygenase produced $59 \mathrm{~g} / \mathrm{L} \mathrm{CCM}$ from catechol through a bioconversion process. ${ }^{6}$ The bioconversion processes rely on the availability of the cheap aromatic precursors, where lignin holds a great potential as the source. Another alternative is to produce CCM de novo from abundant feedstocks, such as glycerol $^{7}$ or sugars. ${ }^{8-10}$

Toward the de novo CCM biosynthesis, several biochemical routes were proposed, all relying on the conversion of shikimate pathway intermediates into PCA or catechol, and finally into CCM. The seven-step shikimate pathway starts with the 3-deoxyarabinoheptulosonate 7-phosphate (DAHP)

Received: November 26, 2019

Published: February 14, 2020 


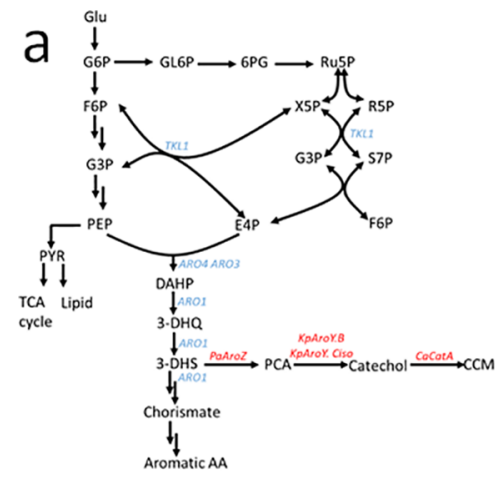

b
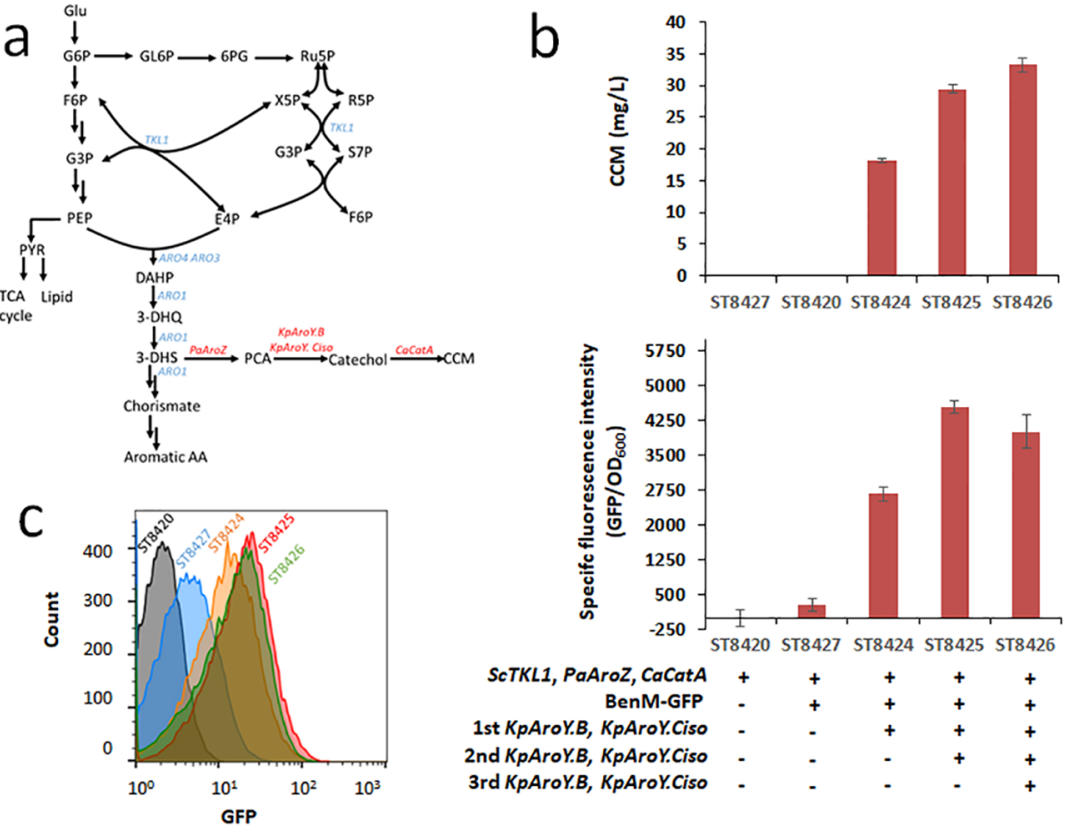

$\begin{array}{rccccc}\text { ScTKL1, PaAroZ, CaCatA } & + & + & + & + & + \\ \text { BenM-GFP } & - & + & + & + & + \\ \text { 1st KpAroY.B, KpAroY.Ciso } & - & - & + & + & + \\ \text { 2nd KpAroY.B, KpAroY.Ciso } & - & - & - & + & + \\ \text { 3rd KpAroY.B, KpAroY.Ciso } & - & - & - & - & +\end{array}$

Figure 1. Biosensor-controlled GFP responds to in vivo cis,cis-muconic acid (CCM) production and enables fluorescence-based selection of improved CCM producing strains. (a) Metabolic pathway for muconic acid biosynthesis in S. cerevisiae. Native enzymes are in blue, heterologous in red. PaAroZ, DHS dehydratase from Podospora anserina; KpAroY.B, KpAroY.Ciso, subunits of functional PCA decarboxylase from Klebsiella pneumoniae; CaCatA, catechol 1,2-dioxygenase from Candida albicans; (b) CCM production and fluorescence output of BenM variant MP02_D04 biosensor response in strains that produce CCM at different levels on YPD medium; (c) flow cytometric analysis of yeast CCM-producing strains implemented with a biosensor on YPD medium. Data shown are mean values \pm SDs of triplicates (b) and one representative of each strains (c). Glu, glucose; G6P, glucose 6-phosphate; GL6P, gluconolactone-6-phosphate; 6PG, gluconate-6-phosphate; Ru5P, ribulose 5-phosphate; X5P, xylulose 5-phosphate; R5P, ribose 5-phosphate; G3P, glyceraldehyde 3-phosphate; S7P, sedoheptulose 7-phosphate; E4P, erythrose 4-phosphate; F6P, fructose 6-phosphate; PEP, phosphoenolpyruvate; PYR, pyruvate; DAHP, 3-deoxyarabinoheptulosonate 7-phosphate; 3-DHQ 3dehydroquinic acid; 3-DHS, 3-dehydroshikimate; PCA, protocatechuic acid; CCM, cis,cis-muconic acid; AA, amino acid.

synthesis from phosphoenolpyruvate (PEP) and erythrose 4phosphate (E4P), and ends with chorismate, the direct precursor of the three aromatic amino acids. Shikimate pathway intermediates, 3-dehydroshikimate (3-DHS) and chorismate, can serve as precursors for CCM biosynthesis (Figure 1a). The route from 3-DHS to CCM comprises three enzymes, DHS dehydratase, PCA decarboxylase, and catechol 1,2-dioxygenase. ${ }^{9,11}$ This is the route that has been most investigated. $^{12-15}$ The alternative routes that start from chorismate, feature three to five enzymatic steps and can go through 4-hydroxybenzoic acid, salicylic acid, or anthranilate as intermediates. $^{8,13,16-18}$ The highest CCM titer from glucose is $59 \mathrm{~g} / \mathrm{L}$, achieved through $88 \mathrm{~h}$ of fermentation of an engineered E. coli strain with boosted 3-DHS supply and expression of downstream pathway genes (DHS dehydratase and PCA decarboxylase from Klebsiella pneumoniae, and catechol 1,2-dioxygenase from Acinetobacter calcoaceticus) inducible by IPTG. ${ }^{19}$ In comparison to CCM production in E. coli, product titers in $S$. cerevisiae strains have so far been significantly lower. Despite this, $S$. cerevisiae serves as a superior CCM production host due to its robust fermentation capabilities, resistance to phage infection, and high protein expression without the need for induction with high-cost inducing agents.

All the works published so far on CCM production in $S$. cerevisiae employed the three-step pathway starting from 3DHS. After implementation of the pathway, the strains were engineered to improve the precursor supply and optimize pathway expression. In particular, the rate-limiting decarboxylation of PCA was relieved by integration of multiple copies of the genes coding for PCA decarboxylase. ${ }^{20}$ Klebsiella pneumonia has three genes that are related to PCA decarboxylase activity, KpAroY.Ciso, KpAroY.B, and KpAroY.D. The first of the genes KpAroY.Ciso encodes the actual PCA decarboxylase, and KpAroY.B encodes a flavin mononucleotide (FMN) prenyltransferase that produces prenylated FMN (prFMN), a cofactor required for the activity of AroY.Ciso. It is not known what the role of KpAroY.D is, but its coexpression with KpAroY.Ciso and KpAroY.B increased CCM production. ${ }^{21} \mathrm{~S}$. cerevisiae has an FMN prenyltransferase encoded by the $P A D 1$ gene. However, in a widely used S. cerevisiae strains of CEN.PK series, $P A D 1$ gene contains a mutation that renders the enzyme inactive, hence expression of $P A D 1$ from another strain, for example, S288c, or KpAroY.B is needed for PCA decarboxylase activity. $^{22}$ Enhanced precursor supply was achieved by rewiring the pentose phosphate pathway ${ }^{23,24}$ or through tailored regulation of the shikimate pathway, including overexpression of feedback-resistant or truncated enzyme variants (Ar$\mathrm{o} 4 \mathrm{p}^{\mathrm{K} 229 L},{ }^{14,23}$ Aro $\left.1 \mathrm{p}^{\Delta \mathrm{E} 9,14}\right)$, and controlled Arolp degradation. ${ }^{15}$ These efforts increased the CCM titer to $c a .1 .2 \mathrm{~g} / \mathrm{L}$ on mineral medium or synthetic defined medium. ${ }^{15,25}$ When amino acids were supplemented, up to $5.1 \mathrm{~g} / \mathrm{L}$ CCM was obtained. $^{15}$

Genetic biosensors are DNA-based units that, in response to in vivo metabolite level (input), generate an output, such as fluorescence, luminescence or controlled gene expression in biological cells. ${ }^{26,27}$ Therefore, these biosensors can be employed for selection of improved microbial metabolite producers through high-throughput screening ${ }^{28}$ or for cell population control. ${ }^{29,30}$ Semirational biosensor-assisted direc- 

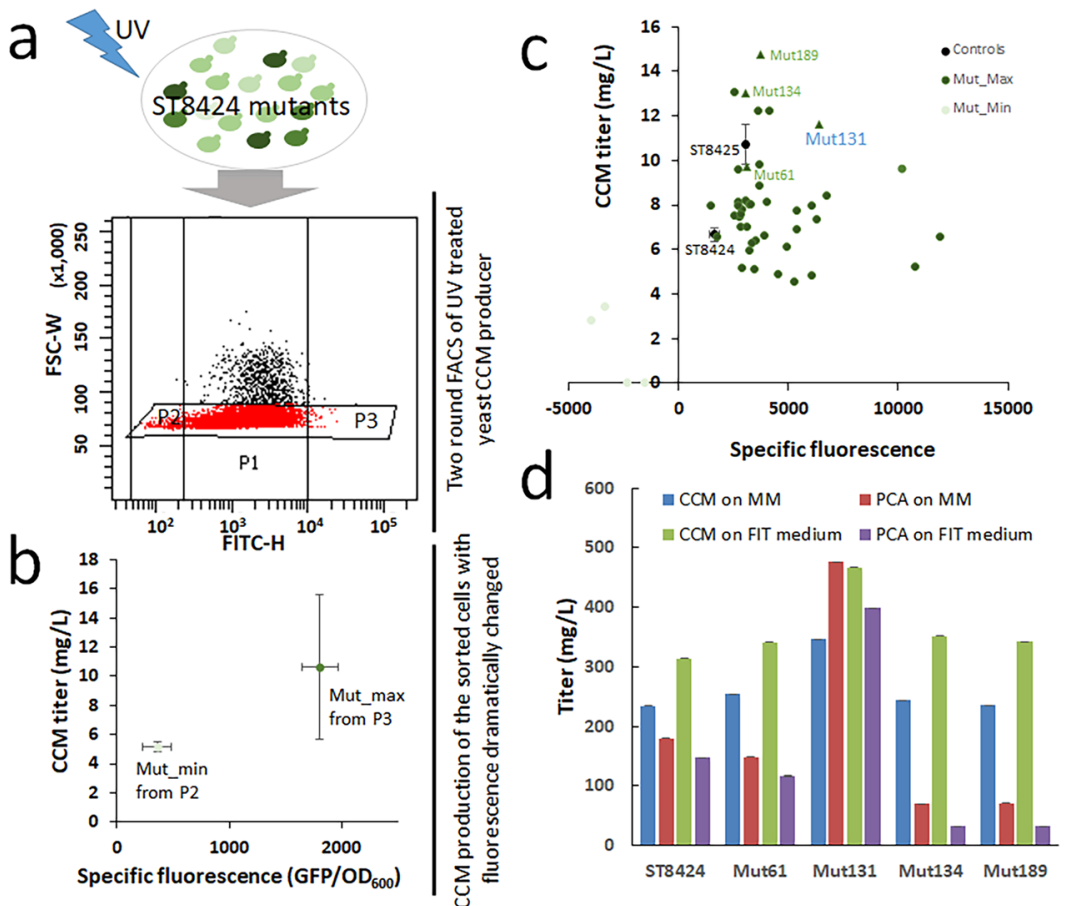

Figure 2. A S. cerevisiae mutant with improved CCM production was identified via biosensor-aided fluorescence-based selection. Ultraviolet (UV) light-treated ST8424 cells were subjected to two rounds of fluorescence activated cell sorting to select the single cells (defined with P1 gate) with the lowest (Min, defined with P2 gate) or highest (Max, defined with P3 gate) fluorescence output (a). CCM production of the sorted cell population (b) and individual cells (c) on YPD medium are evaluated, and potential improved CCM producer strains (marked as triangle) are validated on mineral medium (MM) (d), a mineral medium, and mimicked fed-batch/feed-in-time (FIT) medium (d) with uracil supplementation. All cultivations were performed in batch, and the mimicked glucose-limited fed-batch was realized via an enzymatic digestion of a polysaccharide source, therefore ensuring a continuous glucose feed. Data shown are from single replicates of mutants in panel c, and mean values \pm SDs of triplicates for panel $b$ and $d$ and control strains in panel $c$.

ted evolution has been leveraged to obtain strains with optimized heterologous pathways and increased precursor availability. By using a CCM-responsive transcriptional regulator BenM to drive expression of an antibiotic resistance gene, which renders growth outperformance in the selective condition to the superior CCM producer cells, Snoek et al. were able to isolate $S$. cerevisiae strains with optimized multicopy integration of AroY.B-AroY.Ciso genes. The selected strain produced $\sim 2 \mathrm{~g} / \mathrm{L}$ CCM on mineral medium under fedbatch fermentation. ${ }^{31}$ In another study, Leavitt et al. employed an Aro9p-based biosensor driving expression of antibiotic resistance gene to isolate mutants with improved flux to tyrosine following a two-round adaptive laboratory evolution $(750 \mathrm{~h}+575 \mathrm{~h})$ under Geneticin-containing conditions, and obtained a strain producing $2.1 \mathrm{~g} / \mathrm{L} \mathrm{CCM}$ by redirecting the enhanced flux to the CCM. ${ }^{14}$

Although significant progress has been made in engineering S. cerevisiae for CCM production, the titer, yield, and productivity are still far from commercially relevant levels. Further exploration of $S$. cerevisiae capacity for the production of CCM (and aromatic compounds in general) is therefore needed. To address this goal, we employed a CCM-biosensor variant with a broad response range to screen for improved CCM producing mutants. Through a time-saving screening process via direct fluorescence activated cell sorting (FACS), we identified a mutant that carries multiple mutations for improved CCM and PCA production. This improved strain was then further rationally engineered, resulting in a yeast strain with the highest reported CCM titer, yield, and productivity to date in controlled fed-batch fermentations.

\section{RESULTS}

Validation of Biosensor Response to CCM Production. To identify novel genetic targets for improved CCM production by $S$. cerevisiae, we applied biosensor-based FACS to screen UV-mutagenized producer strains. CCM-producing yeast strains carried a CCM-responsive BenM biosensor ${ }^{20,32}$ to generate fluorescence from single cells directly proportional to the production of CCM. The single cells with higher fluorescence levels (and supposedly higher CCM production) can be selected by FACS.

We started with validating the correlation between the CCM production and fluorescence. The basal strain ST2377 20 was based on CEN.PK102-5B and harbored PaAroZ from Podospora anserina encoding DHS dehydratase, KpAroY.D from $K$. pneumoniae encoding a protein that may improve PCA decarboxylase activity, and CaCatA from Candida albicans encoding catechol 1,2-dioxygenase (Figure 1a). The basal strain did not carry KpAroY.Ciso and KpAroY.B genes from $K$. pneumonia encoding PCA decarboxylase and FMN prenyltransferase, respectively, and without these genes, it could produce PCA, but not catechol or CCM (Figure 1a). ST2377's derivative strains were generated by introducing one, two, or three copies of KpAroY.B and KpAroY.Ciso, genes for the limiting biosynthetic reaction (Figure 1a). The resulting strains were then implemented with a BenM variant MP02_D04, ${ }^{32}$ which shows a broader CCM-sensing range than the original BenM, ${ }^{20}$ to enable a wider detection range and higher resolution for strain screening. By doing so, three strains implemented with the same CCM-biosensor but different copy numbers of KpAroY.B and KpAroY.Ciso, ST8424, ST8425 and 
ST8426, were generated and produced $18.2 \pm 0.3,29.3 \pm 0.7$ and $33.3 \pm 1.1 \mathrm{mg} / \mathrm{L}$ CCM respectively, on complex YPD medium (Figure $1 \mathrm{~b}$ ).

The producing strains had a higher fluorescence than the nonproducing controls in the microplate reader test (Figure 1b) and on flow cytometry (Figure 1c), and the strain with two copies of the pathway (ST8425) could be discriminated from the single-copy strain (ST8424) (Figure 1c). However, two(ST8425) and three-copy strains (ST8426) could not be resolved by flow cytometry. The sensitive response range between ST8424 and ST8425 can be clearly detected with flow cytometry (Figure 1c). In contrast to this, the higher CCM concentration of ST8426 cannot be distinguished from the fluorescence. It is then expected that the improvement in CCM production in ST8424 would result in an easily detectable signal increase, allowing for the subsequent cell sorting. We therefore selected the single-copy strain ST8424 for the UV mutagenesis.

Library Generation and FACS Screening. The strain ST8424 was subjected to UV mutagenesis to create a strain library for screening. The mutants were cultivated and sorted on FACS to select the populations with the high and the low fluorescence output. The high and low-fluorescing populations were cultivated again and sorted on FACS (Figure 2a) to further enrich for correspondingly high- or low-fluorescing cells. The resulting populations were named Mut_max and Mut_min. The populations and individual isolates were cultivated and the fluorescence and CCM production were measured. The mean specific fluorescence was 5-fold higher in culture of Mut_max population than Mut_min population (Figure 2b). The CCM production correlated with fluorescence intensity, with Mut_max pooled cells showing a much higher CCM production (2-fold) compared to the Mut_min pool (Figure 2b).

The fluorescence of 109 out of 270 individual isolates from the Mut_max population was higher than the control ST8424, while that of 81 out of 90 isolates from Mut min was lower (Figure S1a,b). A sampling of 42 isolates from Mut max showing $>25 \%$ increase in the specific fluorescence and four isolates from Mut_min showing $>70 \%$ reduction in the specific fluorescence (Figure S1c) were selected for the CCM production. Twenty-six of the 42 Mut max strains produced more CCM on YPD medium than the control ST8424 (up to a 96.3\% improvement) (Figure $2 \mathrm{c}$ and Figure S2), whereas all four Mut_min strains produced less CCM (Figure 2c). These results demonstrated that the BenM variant MP02 D04 can be reliably utilized using FACS to isolate improved yeast CCM producing variants.

To test the robustness of the improved CCM producing mutants, we further tested CCM production on alternative media, mineral medium (MM), and simulated fed-batch medium (Feed-In-Time, FIT). The MM support the cell growth through de novo synthesis of the metabolites and may therefore supply more precursors for biosynthesis of aromatic metabolites in comparison to that on YPD, which contains compounds that feedback-inhibit the biosynthetic pathway for aromatics. The FIT medium is a modified MM, in which the glucose is continuously released from a polysaccharide source at a low level. The use of FIT medium therefore limits the overflow metabolism and allows a mimicking of a glucoselimited fed-batch cultivation in the small scale cultivation. By using this setup, the potential of the strain for the CCM production in the large scale fermentation can be evaluated.
Nine out of forty-two Mut max strains, with a greater than $30 \%$ increase in both $\mathrm{CC} \overline{\mathrm{M}}$ titer and $\mathrm{CCM}$ yield $(\mathrm{mg} / \mathrm{L} /$ $\mathrm{OD}_{600}$ ) on YPD medium (Figure $2 \mathrm{c}$ and Figure S2) were selected for analysis. The strains showed improvement in CCM production up to $49.7 \%$ on MM or FIT medium (Figure $2 \mathrm{~d}$ and Figure S3), with the best producer Mut131 showing a $74.4 \%, 49.3 \%$, and $49.7 \%$ improvement in CCM production compared to the control strain on YPD, MM, and FIT medium, respectively (Figure $2 \mathrm{c}, \mathrm{d}$ ). The highest titer of 470.6 $\mathrm{mg} / \mathrm{L}$ was achieved on FIT medium (Figure $2 \mathrm{~d}$ ). Interestingly, the Mut131 also produced more PCA, the precursor of CCM, than the control strain ST8424, showing a $164 \%$ and $170 \%$ increase on MM and FIT medium, respectively (Figure 2d). These results suggested that the Mut131 strain, identified through the BenM variant MP02 D04-mediated FACS, was a variant strain robust in improved CCM production.

Identification of Causal Mutations by Reverse Engineering. To decipher the genetic determinants for improved CCM and PCA production in Mut131, we resequenced the genome and identified 25 and 9 mutations in native gene coding sequences (CDS) (Table 1) and in

Table 1. Mutations Identified in Mut131 through Genome Resequencing

\begin{tabular}{|c|c|c|}
\hline mutation type & mutated gene & specific mutation \\
\hline missense & PWP2 & Ser388Phe \\
\hline missense & EST2 & Val182Met \\
\hline missense & RET2 & Pro15Ser \\
\hline missense & IMA3 & Asp242Asn \\
\hline missense & ATG1 & Glu382Lys \\
\hline missense & PET54 & Gly19Glu \\
\hline missense & OCA5 & Ala649Val \\
\hline missense & DIT1 & Glu526Gly \\
\hline missense & $S A P 1$ & Pro20Leu \\
\hline missense & PUT3 & Ser615Phe \\
\hline missense & NUD1 & Arg819Trp \\
\hline missense & KNS1 & Ser227Leu \\
\hline missense & $\mathrm{CDC} 15$ & Pro429Phe \\
\hline missense & CTS2 & Gly497Glu \\
\hline missense & MNE1 & Lys450Glu \\
\hline nonsense & NUP53 & Ser69* \\
\hline silent & $R P P 2 B$ & Ser29Ser \\
\hline silent & MRPS18 & Ser52Ser \\
\hline silent & SIP2 & Leu381Leu \\
\hline silent & $M L P 1$ & Leu661Leu \\
\hline silent & YLR050C & Leu112Leu \\
\hline silent & DUG1 & Phe342Phe \\
\hline silent & VAC14 & Gly474Gly \\
\hline silent & MET22 & Asp342Asp \\
\hline silent & $B U B 1$ & Leu274Leu \\
\hline duplication & PaAroZ-CaCatA-KlLEU2 & NA \\
\hline
\end{tabular}

intergenic regions (Table S1), respectively. Of the 25 mutations, 15 were missense mutations, 1 was a nonsense mutation, and 9 were silent mutations (Table 1). No duplications of the native genome regions were detected, but a duplication of the heterologous genes PaAroZ-CaCatAKlLEU2 (Table 1) was found (Figure S4).

We first sought to experimentally validate the effect of the single mutations on the CCM production. All missense and nonsense mutations, except $I M A 3$, were reintroduced into the ST8424 parental strain individually. IMA3 was excluded from 

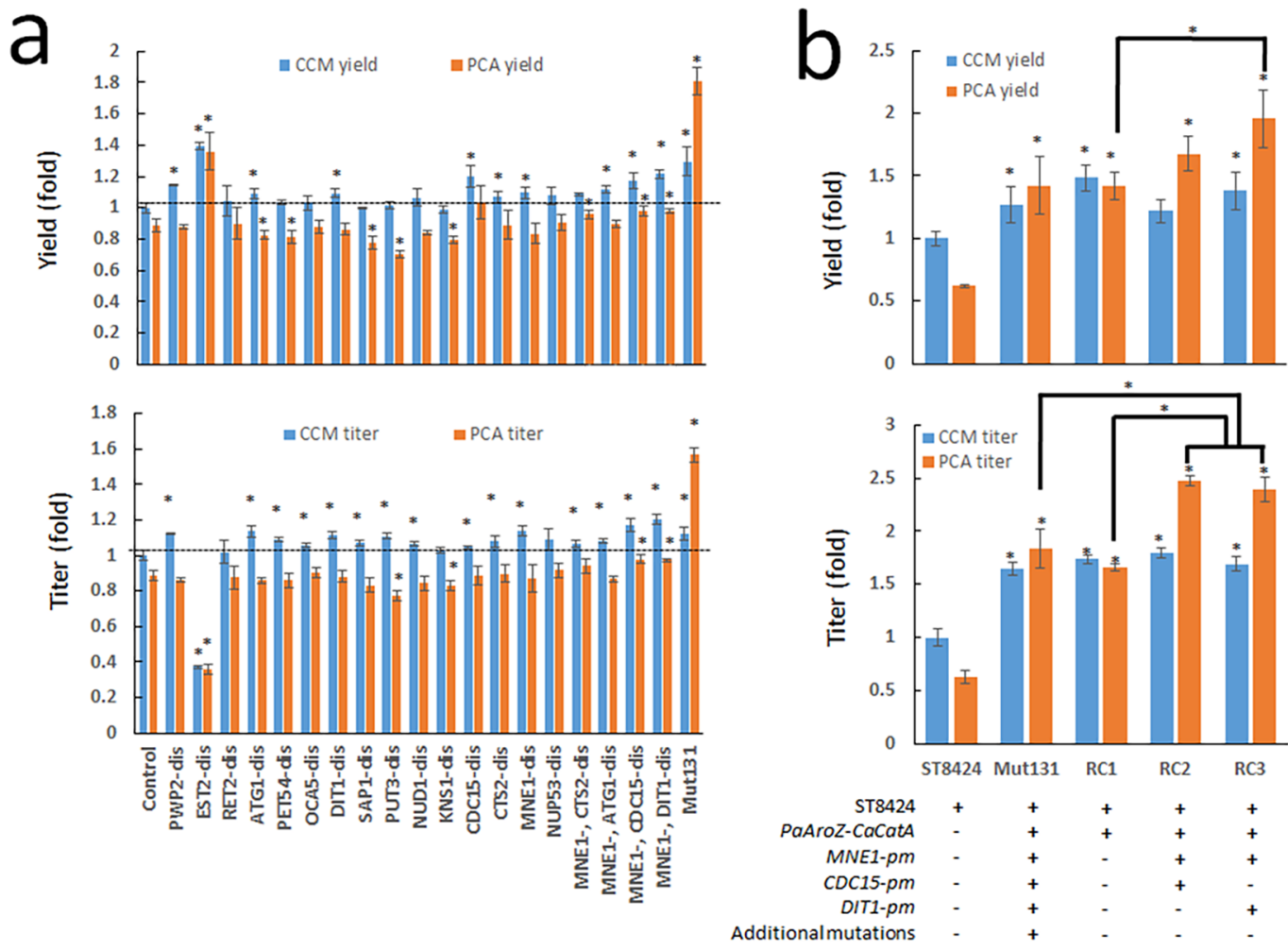

Figure 3. Reverse engineering of identified mutations for improved CCM production. All tests were performed on $72 \mathrm{~h}$ culture on mineral medium without uracil (a) or supplemented with $20 \mathrm{mg} / \mathrm{L}$ uracil (b). The specific yield $\left(\mathrm{mg} / \mathrm{L} / \mathrm{OD}_{600}\right)$ and titer are shown. The CCM and PCA values for mutants, are all shown as fold changes in relation to the CCM values of the control strains. Data shown are mean values \pm SDs of triplicate. Statistical difference between control and indicated strains ( $a$ and $b$ ), as well as that between the indicated strains ( $b$ ) was determined by two-tailed Student's $t$ test $\left(*_{p}<0.05\right)$. Gene-dis, strain carrying indicated gene $(\mathrm{s})$ with a designed point mutation; pm, point mutation.

the reverse engineering as it is functionally redundant to multiple genes of the isomaltase family (IMA1, IMA2, IMA4). All the mutations were generated via the CRISPR-Cas9 method, using a URA3 plasmid harboring both a guide RNA cassette and the donor fragment for mutation introduction.

Both CCM yield $\left(\mathrm{mg} / \mathrm{L} / \mathrm{OD}_{600}\right)$ and CCM titer were evaluated to account for the potential growth defect of mutations. The Mut131 strain harboring a URA3 empty vector showed $29.4 \pm 9.1 \%$ and $12.1 \pm 3.7 \%$ increase in the CCM yield and CCM titer, respectively, compared to the ST8424 control strain harboring a URA3 empty vector (Figure 3a). While most reintroduced single mutations resulted in a higher CCM titer (up to $13.7 \pm 2.6 \%$ in MNE1 mutated strain (MNE1-dis)), only seven mutations (PWP2, EST2, ATG1, DIT1, CDC15, CTS2, and MNE1) significantly improved the CCM yield, with a maximum improvement of $39.5 \pm 2.4 \%$ and $20.1 \pm 6.8 \%$ for EST2-dis and CDC15-dis strains (Figure 3a). However, none of the single mutations resulted in improved CCM production equivalent to the Mut131 strain. Furthermore, some mutations resulted in significantly lower biomass formation, with EST2-dis and CDC15-dis strains showing a $63.0 \pm 0.6 \%$ decrease and $4.6 \pm 0.7 \%$ increase in CCM titer, respectively. A combinatorial effect of all the mutations was thus speculated as associated with the improvement in Mut131.

To verify the cumulative effect of the mutations, we made double mutants, by introducing mutations in CTS2, ATG1, CDC15, or DIT1 into the MNE1-dis strain. While CTS2- and ATG1-dis strains did not show any improvement in combination with $M N E 1-\mathrm{pm}$ (point mutation), CDC15-pm and DIT1-pm combined with MNE1-pm enhanced both CCM yield and CCM titer, with a $20.5 \pm 2.7 \%$ (CCM yield) and $21.5 \pm 3.0 \%$ (CCM titer) increase (compared to control strain) (Figure 3a). This combination of two mutations, CDC15-MNE1-pm or DIT1-MNE1-pm, completely restored the CCM production phenotype of Mut131 in both CCM titer and yield; however, the increase in the PCA production $(9.8 \pm$ $0.9 \%)$ was still smaller than that of Mut131 (76.9 $\pm 4.6 \%)$ (Figure 3a). These results suggested that individual and cumulative beneficial mutations were partially characterized for the improvement of PCA and CCM production in the Mut131.

We further tested the contribution of the duplication of $\mathrm{PaAroZ}$ and $\mathrm{CaCatA}$, by introducing an additional copy of the expression cassettes into the ST8424 parental strain. Surprisingly, the overexpression of these two genes responsible for the biosynthetic steps that were not recognized as fluxcontrolling before, increased the yield and titer of both CCM and PCA to that of the mutant (RC1 vs Mut131) (Figure 3b). Moreover, the combination of the gene duplication and beneficial double mutations gave a much higher PCA production and a comparable CCM production (Figure $3 \mathrm{~b}$ ), with a $49.0 \pm 3.1 \%$ higher titer (RC2 that harbors MNE1CDC15-pm vs RC1) and a $37.6 \pm 1.7 \%$ higher PCA yield (RC3 that harbors MNE1-DIT1-pm vs RC1) achieved (Figure $3 \mathrm{~b}$ ). RC2 and RC3 also produced up to $34.7 \%$ more PCA (titer) than Mut131 (Figure 3b), even though no improvement in the PCA yield was observed. These results indicated that the improved CCM and PCA in Mut131 derived from the duplication of $\mathrm{PaAroZ}$ and $\mathrm{CaCatA}$ (main factor) as 


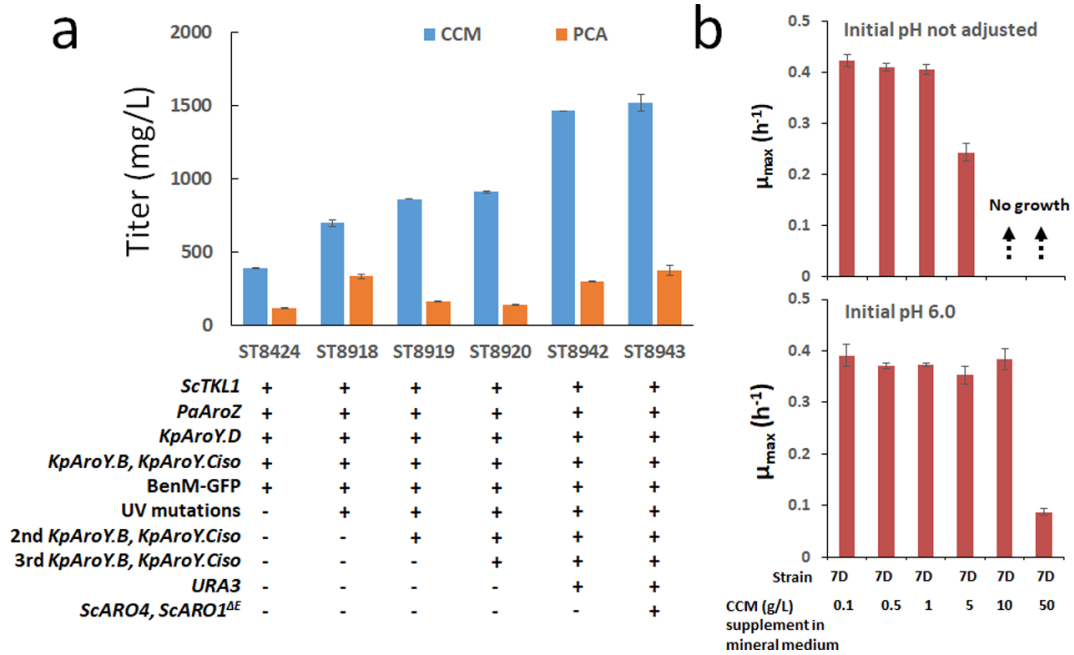

Figure 4. CCM production of rationally engineered strains (a) and CCM toxicity to S. cerevisiae strain CEN.PK113-7D (b). (a) CCM production of engineered strains on FIT medium with (ST8424, ST8918, ST8919, ST8920) or without uracil supplementation (ST8942, ST8943) for 72h. (b) Maximum specific growth rate of CEN.PK113-7D on mineral medium with CCM supplementation and initial pH unadjusted or adjusted. In tests with $\geq 5 \mathrm{~g} / \mathrm{L}$ CCM supplementation and initial $\mathrm{pH}$ unadjusted, CCM is largely in the insoluble form, whereas, it is all soluble in the remainder of the tests. Data shown are mean values \pm SDs of triplicates.

well as the mutations, where both beneficial and detrimental mutations take effect together.

Rational Metabolic Engineering of the Mutant Strain. In parallel to the reverse engineering work, we implemented several rational metabolic engineering strategies into the Mut131 strain (further named as ST8918) for higher CCM production. Specifically, we introduced additional copies of the CCM biosynthetic enzymes, restored URA3 prototrophy, and improved the precursor supply. Introduction of the second and third copies of the expression cassettes for KpAroY.B and KpAroY.Ciso improved the conversion of PCA to CCM. In strain ST8920, with three copies of KpAroY.B and KpAroY.Ciso genes, the titer of CCM was $908 \mathrm{mg} / \mathrm{L}$ and PCA $139 \mathrm{mg} / \mathrm{L}$ (Figure 4a). This represents an improvement in the CCM titer of $30.7 \%$ to ST8918. The total amount of CCM and PCA produced remained unchanged, ca. $1 \mathrm{~g} / \mathrm{L}$ (Table S2). The restoration of uracil prototrophy resulted in further $61.2 \%$ increase in CCM titer of $1.46 \mathrm{~g} / \mathrm{L}$ (Figure $4 \mathrm{a}$ ). The combined PCA and CCM titer increased to $1.76 \mathrm{~g} / \mathrm{L}$ (Table S2). To validate the involvement of URA3 and uracil metabolism in CCM production, a uracil supplementation test was performed on two ura3- strains with distinct CCM production capabilities, the original strain ST8424 and mutatedengineered strain ST8920 (Figure S5). Results showed that CCM was produced in a uracil-dependent pattern in both strains (Figure S5), which verified the contribution of uracil metabolism to CCM aside from an improvement in cell growth. Finally, precursor supply was increased by additionally expressing an AROM protein without shikimate dehydrogenase domain Arolp ${ }^{\Delta \mathrm{E}}$ and DAHP synthase Aro4p under strong constitutive promoters PGK $1 \mathrm{p}$ and TEF $1 \mathrm{p}$, respectively. The resulting strain ST8943 produced up to $1.59 \mathrm{~g} / \mathrm{L} \mathrm{CCM}$ and $0.42 \mathrm{~g} / \mathrm{L}$ PCA in a deep-well plate assay on FIT medium (Figure 4a).

High-level production of CCM may result in toxic effects to the cell and could retard cell growth thereby resulting in lower overall CCM production. Indeed, we observed a decrease in the biomass accumulation in strains with increased CCM production (ST8918, ST8919, and ST8920) (Figure S6). To investigate whether CCM was toxic to $S$. cerevisiae cells particularly at high concentrations, the reference strain CEN.PK 113-7D was exposed to increasing CCM levels, and the growth was monitored. Since CCM exists in either protonated or unprotonated forms, depending on the $\mathrm{pH}$, we tested the toxicity with or without adjustment of the initial $\mathrm{pH}$ of the medium. When $\mathrm{pH}$ was not adjusted after the addition of CCM, a $43 \%$ reduction in the maximum specific growth rate was observed in the presence of $5 \mathrm{~g} / \mathrm{L} \mathrm{CCM}$, and cell growth was completely abolished at $10 \mathrm{~g} / \mathrm{L}$ (Figure $4 \mathrm{~b}$ and Figure $\mathrm{S} 7 \mathrm{a}$ ). When the initial $\mathrm{pH}$ adjusted to 6.0 after the addition to CCM, the cells could tolerate much higher CCM concentrations. The growth inhibition, indicated by a significant decrease in both maximum specific growth rate $(78 \%$, Figure $4 \mathrm{~b})$ and biomass accumulation (69\%, Figure S7b) was only observed with $50 \mathrm{~g} / \mathrm{L}$ CCM, even though a $17-42 \%$ reduction in the final biomass accumulation occurred at $5 \mathrm{~g} / \mathrm{L}$ and $10 \mathrm{~g} /$ L CCM (Figure S7b). In addition, the intracellular CCM concentration of cells exposed to these conditions (showing growth inhibition) was comparable to the intracellular concentration measured in the engineered CCM producer strains (ST8424 and ST8920) (Figure S8), indicating that CCM is produced at a high enough level to cause cellular toxicity. Furthermore, the $\mathrm{pH}$ of the culture showing over $43 \%$ growth inhibition was still relatively high (3.43) at the end of the cultivation (Figure S7c), verifying that the main inhibitory effect on cell growth is from CCM toxicity instead of the low $\mathrm{pH}$. These data altogether indicate that CCM toxicity is $\mathrm{pH} /$ protonated form dependent and without $\mathrm{pH}$ control, $5 \mathrm{~g} / \mathrm{L}$ CCM is sufficiently toxic to $S$. cerevisiae.

Fed-Batch Fermentation in Bioreactors. To evaluate the CCM production by the engineered strain ST8943, we performed fed-batch fermentations in controlled bioreactors. The fermentation was carried out on a mineral medium with the vitamin composites substituted by yeast extract. In industrial-scale fermentations, it is preferably to use yeast extract as a vitamin source instead of a defined vitamin mix because of lower cost and a simpler handing as yeast extract can be autoclaved. Throughout the fed-batch phase, glucose 

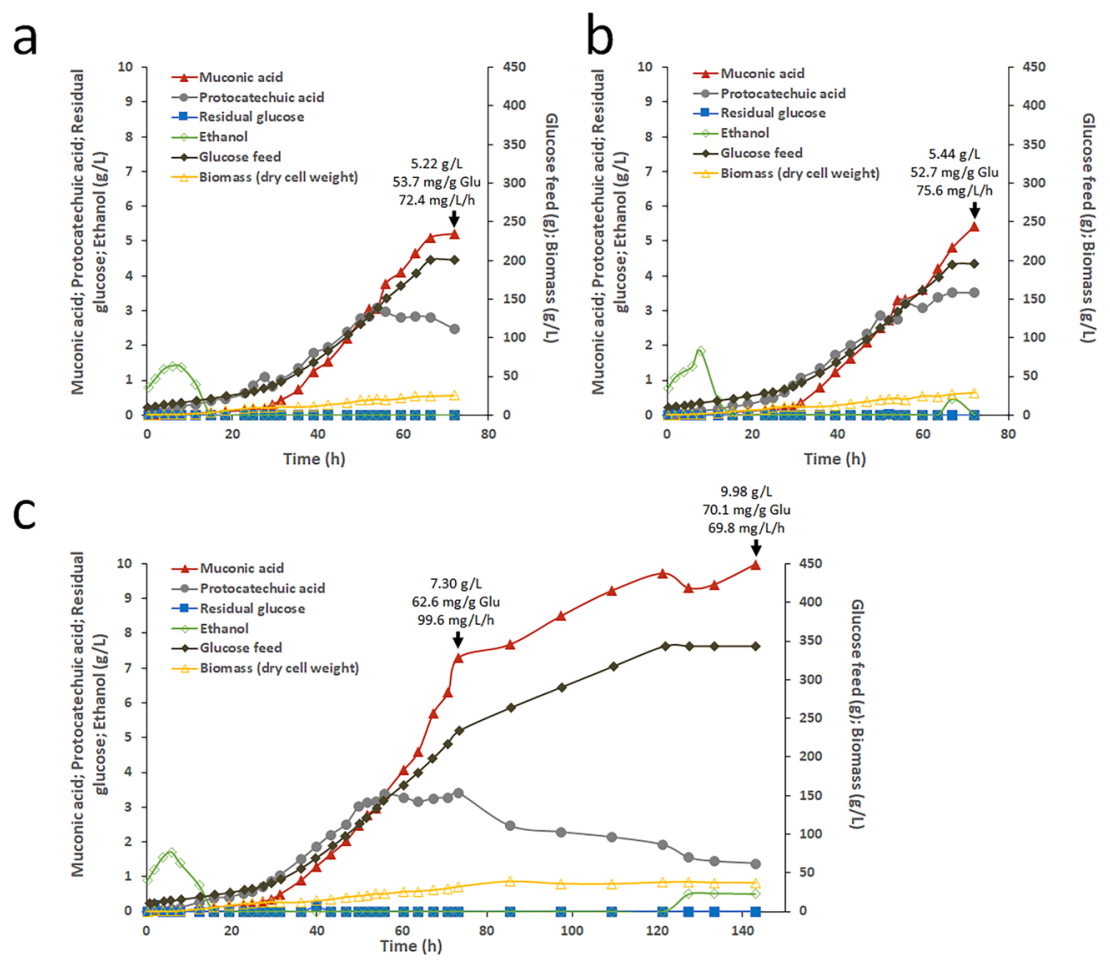

Figure 5. Controlled fed-batch fermentation for CCM production. Fermentations BD3 (a), BD4 (b), and BD5 (c) of ST8943 strain were performed with different feed solutions. The feed solution for BD4 contained additional salt, yeast extract, and trace metal compared to that of BD3. Feed solution for BD5 contained all components for BD4 except yeast extract. CCM titers (g/L), yields (mg/g glucose (Glu)), and productivities $(\mathrm{mg} / \mathrm{L} / \mathrm{h})$ indicated were for $72 \mathrm{~h}(\mathrm{a}), 72 \mathrm{~h}(\mathrm{~b}), 73 \mathrm{~h}(\mathrm{c})$, and $143 \mathrm{~h}(\mathrm{c})$, respectively. Data shown are from a single replicate.

Table 2. Comparison of Titer, Yield, and Productivity of Reported S. cerevisiae CCM Cell Factories

\begin{tabular}{|c|c|c|c|c|c|}
\hline $\begin{array}{l}\text { titer } \\
(\mathrm{g} / \mathrm{L})\end{array}$ & $\begin{array}{c}\text { yield } \\
(\mathrm{mg} / \mathrm{g} \text { sugar })\end{array}$ & $\begin{array}{l}\text { volumetric productivity } \\
\qquad(\mathrm{mg} / \mathrm{L} / \mathrm{h})\end{array}$ & medium & cultivation & ref \\
\hline 0.00156 & 0.078 & 0.0092 & synthetic complex medium & shake flask/batch & 9 \\
\hline 0.141 & 3.53 & 2.94 & synthetic complex medium & shake flask/batch & 23 \\
\hline 0.32 & 8 & 4.45 & mineral medium & shake flask/batch & 24 \\
\hline 2.1 & 12.9 & 8.75 & synthetic complex medium & $\begin{array}{l}\text { bioreactor/fed- } \\
\text { batch }\end{array}$ & 14 \\
\hline 1.244 & 31 & 10.37 & synthetic complex medium & shake flask/batch & 25 \\
\hline 1.927 & 11.4 & 23.8 & synthetic complex medium & $\begin{array}{l}\text { bioreactor/fed- } \\
\text { batch }\end{array}$ & 31 \\
\hline 2.008 & 13.4 & 19.9 & synthetic complex medium & $\begin{array}{l}\text { bioreactor/fed- } \\
\text { batch }\end{array}$ & 31 \\
\hline 1.2 & NA & 7.14 & mineral medium & $\begin{array}{l}\text { bioreactor/fed- } \\
\text { batch }\end{array}$ & 15 \\
\hline 5.1 & 58 & 30.36 & mineral medium supplemented with amino acid & $\begin{array}{l}\text { bioreactor/fed- } \\
\text { batch }\end{array}$ & 15 \\
\hline 7.55 & 45.8 & 138 & $\begin{array}{l}\text { mineral medium with vitamin substituted by yeast } \\
\text { extract }\end{array}$ & $\begin{array}{l}\text { bioreactor/fed- } \\
\text { batch }\end{array}$ & this study $\left(\mathrm{BD} 2^{a}\right)$ \\
\hline 9.98 & 70.1 & 69.8 & $\begin{array}{l}\text { mineral medium with vitamin substituted by yeast } \\
\text { extract }\end{array}$ & $\begin{array}{l}\text { bioreactor/fed- } \\
\text { batch }\end{array}$ & this study $\left(\mathrm{BDS}^{a}\right)$ \\
\hline 20.8 & 66.2 & 139 & $\begin{array}{l}\text { mineral medium with vitamin substituted by yeast } \\
\text { extract }\end{array}$ & $\begin{array}{l}\text { bioreactor/fed- } \\
\text { batch }\end{array}$ & this study $\left(\mathrm{BD}^{b}\right)$ \\
\hline
\end{tabular}

${ }^{a}$ In fermentation BD5, glucose feeding rate was lower, nutrient supplemented in feed solution was more abundant, and fermentation was performed longer $\left(143 \mathrm{~h}\right.$ vs $54.6 \mathrm{~h}$ ) compared to BD2. ${ }^{b}$ In fermentation BD6, glucose feeding rate was even lower in the first $24 \mathrm{~h}$ compared to BD5. Data shown is from $149.5 \mathrm{~h}$. Yeast extract was only present in the starting medium instead of the feed solution in fermentation BD2, BD5 and BD6.

concentration was maintained below $1 \mathrm{~g} / \mathrm{L}$ (mostly $0 \mathrm{~g} / \mathrm{L}$ ) to avoid the Crabtree effect and overflow metabolism (Figure 5 and Figure S9). The $\mathrm{pH}$ was maintained at $6.0 \pm 0.1$ to minimize the toxicity of CCM (Figures S10 and S11).

A first set of two short fermentations $(54.6 \mathrm{~h})$ were performed (BD1 and BD2), both comprising an initial batch phase followed by a fed-batch phase. The feed solution contained $800 \mathrm{~g} / \mathrm{L}$ glucose. The feed solution for BD2 additionally contained $4 \mathrm{~g} / \mathrm{L} \quad \mathrm{KH}_{2} \mathrm{PO}_{4}$. A growth-coupled production pattern was observed for CCM and PCA production (Figure S9). BD1 fermentation resulted in 38.3 $\mathrm{g} / \mathrm{L}$ dry cell weight and in product concentrations of $6.18 \mathrm{~g} / \mathrm{L}$ for CCM and $4.05 \mathrm{~g} / \mathrm{L}$ for PCA (Figure S9a). In the fermentation $\mathrm{BD} 2$ with $\mathrm{KH}_{2} \mathrm{PO}_{4}$ supplementation, the biomass 
concentration was higher $(51.2 \mathrm{~g} / \mathrm{L}$ dry cell weight) and product titers were slightly higher, $7.55 \mathrm{~g} / \mathrm{L} \mathrm{CCM}$ and $4.25 \mathrm{~g} /$ $\mathrm{L}$ PCA (Figure S9b). In these processes, up to $6.45 \mathrm{~g} / \mathrm{L}$ (Figure S9a) and $5.76 \mathrm{~g} / \mathrm{L}$ (Figure S9b) ethanol was accumulated at the early stage, and limited salt concentration $\left(\mathrm{Mg}^{2+}\right.$ and $\left.\mathrm{PO}_{4}{ }^{3-}\right)$ was observed at the late stage (Figure S10).

To optimize the fermentation process with the purpose of lowering the overflow metabolism and ensure sufficient nutrients for the cell growth, we sought to start the cultivation with glucose-free medium in the bioreactor and to reduce the glucose feeding rate (Figure 5) using a solution with $200 \mathrm{~g} / \mathrm{L}$ glucose (the concentration was reduced in order to allow for the low glucose feed rate with the available pumps, with minimum feed rates of about $3 \mathrm{~mL} / \mathrm{h}$ ), $15 \mathrm{~g} / \mathrm{L} \mathrm{KH}_{2} \mathrm{PO}_{4}$, and 8 $\mathrm{g} / \mathrm{L} \mathrm{MgSO}_{4}$ (BD3, Figure 5a, Figure S11). Two additional fermentation runs (BD4 and BD5) were similarly designed, with the exception of the feed composition. The feed solutions for BD4 and BD5 contained additional amounts of $\left(\mathrm{NH}_{4}\right)_{2} \mathrm{SO}_{4}$, yeast extract, $\mathrm{CaCl}_{2}$, and trace metals (BD4, Figure $5 b$, Figure $\mathrm{S} 11)$ or $\left(\mathrm{NH}_{4}\right)_{2} \mathrm{SO}_{4}, \mathrm{CaCl}_{2}$, and trace metals (BD5, Figure 5c, Figure S11), respectively. With the optimization, the high ethanol accumulation in the early fermentation stage (up to $6.45 \mathrm{~g} / \mathrm{L}, \mathrm{BD} 1$, Figure S9a) was reduced to up to 1.41 (BD3, Figure 5a), 1.83 (BD4, Figure $5 \mathrm{~b}$ ), and $1.56 \mathrm{~g} / \mathrm{L}$ (BD5, Figure 5c), respectively. As well, the CCM yield ( $\mathrm{mg} / \mathrm{g}$ glucose) was improved significantly when ca. $200 \mathrm{~g}$ of glucose was fed, from 39.4 ( $47 \mathrm{~h}$ in BD1, Figure S9a), 43.4 ( $47 \mathrm{~h}$ in BD2, Figure S9b) to 53.7 ( $72 \mathrm{~h}$ in BD3, Figure $5 \mathrm{a}), 52.7$ ( $72 \mathrm{~h}$ in $\mathrm{BD} 4$, Figure $5 \mathrm{~b})$, and $55.1 \mathrm{mg} / \mathrm{g}$ glucose $(67 \mathrm{~h}$ in BD5, Figure 5c). Since the use of feed solution with lower glucose concentration (200 vs $800 \mathrm{~g} / \mathrm{L}$ ) gave a $c$ a. $40 \%$ higher broth volume, a relatively lower final titer was achieved, resulting in $5.22 \mathrm{~g} / \mathrm{L} \mathrm{CCM}$ and $2.49 \mathrm{~g} / \mathrm{L}$ PCA (BD3, Figure 5a), $5.44 \mathrm{~g} / \mathrm{L} \mathrm{CCM}$ and $3.52 \mathrm{~g} / \mathrm{L} \mathrm{PCA} \mathrm{(BD4,}$ Figure $5 \mathrm{~b}$ ), and $7.30 \mathrm{~g} / \mathrm{L} \mathrm{CCM}$ and $3.40 \mathrm{~g} / \mathrm{L} \mathrm{PCA} \mathrm{(BD5,}$ Figure 5c) after $72 \mathrm{~h}$ (BD3 and BD4) or $73 \mathrm{~h}$ (BD5) cultivation. However, after $143 \mathrm{~h}$ fermentation in BD5, a high titer of $9.98 \mathrm{~g} / \mathrm{L} \mathrm{CCM}$ and $1.38 \mathrm{~g} / \mathrm{L}$ PCA was realized, with the yield of $70.1 \mathrm{mg} / \mathrm{g}$ glucose and the productivity of 69.8 $\mathrm{mg} / \mathrm{L} / \mathrm{h}$ (Figure $5 \mathrm{c}$ and Table 2).

Despite the high CCM production in BD5, the biomass accumulation at the late stage $(85.3-143 \mathrm{~h})$ stopped, while CCM concentration only slowly increased. We then sought to further optimize the fermentation process of BD5 by increasing the feeding rate for the late stage (after $73.5 \mathrm{~h}$ ) to support more biomass accumulation for CCM production. This optimization enabled a much higher biomass accumulation, from $36.8 \mathrm{~g} / \mathrm{L}(\mathrm{BD} 5$ at $143 \mathrm{~h}$ ) to $69 \mathrm{~g} / \mathrm{L}$ dry cell weight (BD6 at $143.5 \mathrm{~h}$ ) (Figure 6), requiring agitation at the maximum speed $(1200 \mathrm{rpm})$ from $121.5 \mathrm{~h}$ (Figure S12). A high titer of $20.8 \mathrm{~g} / \mathrm{L}$ CCM and $1.91 \mathrm{~g} / \mathrm{L}$ PCA was achieved after $149.5 \mathrm{~h}$ fermentation in BD6, with the yield of $66.2 \mathrm{mg} / \mathrm{g}$ glucose and the productivity of $139 \mathrm{mg} / \mathrm{L} / \mathrm{h}$ (Figure 6). The titer, yield, and productivity represent a significant improvement compared to the previous reports (Table 2).

\section{DISCUSSION}

In this work, we leveraged the power of a biosensor-based selection to discover new nonintuitive metabolic engineering targets for improvement of CCM production in S. cerevisiae (Figure 1). An improved mutant strain Mut131, which harbored duplication of genes for CCM biosynthesis and novel causal mutations unrelated to the known pathways to

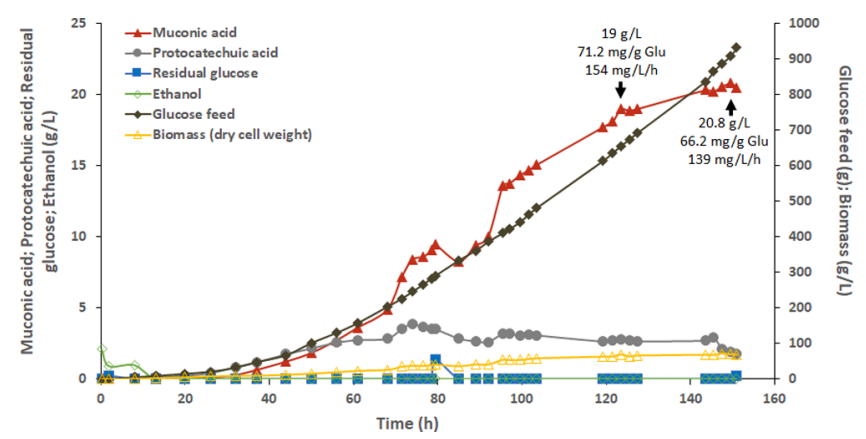

Figure 6. Controlled fed-batch fermentation for CCM production. Fermentation BD6 of ST8943 strain was performed by feeding with $465.32 \mathrm{~mL}$ of $1 \times$ feed solution $\left(200 \mathrm{~g} / \mathrm{L}\right.$ glucose, $15 \mathrm{~g} / \mathrm{L} \mathrm{KH}_{2} \mathrm{PO}_{4}, 8$ $\mathrm{g} / \mathrm{L} \mathrm{MgSO}_{4}, 10 \mathrm{~g} / \mathrm{L}\left(\mathrm{NH}_{4}\right)_{2} \mathrm{SO}_{4}, 0.4 \mathrm{~g} / \mathrm{L} \mathrm{CaCl}_{2}$, and trace metal $)$ and $1399 \mathrm{~mL}$ of $3 \times$ feed solution into $1.3 \mathrm{~L}$ starting fermentation broth during $0-48 \mathrm{~h}$ and $48-151 \mathrm{~h}$, respectively. CCM titers $(\mathrm{g} / \mathrm{L})$, yields $(\mathrm{mg} / \mathrm{g}$ glucose $(\mathrm{Glu}))$ and productivities $(\mathrm{mg} / \mathrm{L} / \mathrm{h})$ indicated were for 123.5 and $149.5 \mathrm{~h}$, respectively. These two time-points correspond to the highest yield/productivity and titer achieved in this run. Data shown are from a single replicate.

CCM (including glycolysis, pentose phosphate pathway, and shikimate pathway) (Table 1 and Figure 3), was isolated, with $49 \%$ higher CCM and 164\% higher PCA titers (Figure 2). This mutant strain was then further rationally engineered (Figure 4) resulting in the production of up to $20.8 \mathrm{~g} / \mathrm{L}$ CCM (Figure 6), in controlled fed-batch fermentations, where CCM toxicity was minimized.

FACS was employed in this work, instead of biosensorassisted strain evolution, as the screening methodology is more direct and significantly shorter in time. In contrast to cultivations lasting up to $1324 \mathrm{~h},{ }^{14,31}$ only two short rounds of cultivation ( $24 \mathrm{~h}$ each) were performed to propagate the cells for the strain screening process that takes hours. Furthermore, a BenM biosensor variant with a broader CCM response range ${ }^{32}$ was employed to avoid fluorescence signal saturation at low in vivo CCM levels, which more likely allowed for the identification of a larger number of high CCM producers.

The identified mutant strain demonstrated significantly improved PCA and CCM production. The improvement mainly resulted from the duplication of genes for DHS dehydratase and catechol 1,2-dioxygenase, indicating that these reactions are also flux-controlling along with the PCA decarboxylation step. ${ }^{20}$

Several missense mutations identified also increase the production of PCA and/or CCM; however, none of these mutations were related to the known precursor supply pathways. This highlights the complexity of the S. cerevisiae cellular machinery associated to the shikimate pathway, as well as the possibility to engineer other nonintuitive targets for further increasing CCM precursor supply, and possibly also for other shikimate pathway associated compounds.

The identified mutations in Mut131 likely impact CCM production in a combinatorial way, as some individual mutations (EST2 and CDC15) significantly reduced the cell growth while the Mut131 strain with all mutations showed no growth defect, as well as the cumulative effect from the combination of mutations (Figure 3a) (and gene duplication (Figure $3 \mathrm{~b})$ ). The nonintuitive beneficial single mutations resulting in an improvement in CCM production are involved in diverse biological processes. PWP2 encodes a $90 \mathrm{~S}$ pre- 
ribosomal component, ${ }^{33}$ EST2 is a reverse transcriptase subunit of the telomerase holoenzyme, ${ }^{34}$ CTS2 is a putative chitinase, ${ }^{35}$ and MNE1 participates in COX1 mRNA splicing. ${ }^{36}$ Although the reported functions of these genes fail to account for the improved CCM production, additional as yet unknown cellular functions may be explored further for the purpose of deeply understanding cellular machinery. This is supported by a previous report on $M N E 1$, in which an MNE1 mutant strain grew substantially better anaerobically and had a higher specific ethanol production under aerobic conditions. ${ }^{37}$ Beneficial mutations in ATG1 and CDC15, two protein kinases required for autophagy induction/cell cycle progression ${ }^{38,39}$ and mitotic exit network ${ }^{40}$ respectively, indicate a possible connection to cellular metabolite turnover and cell division. The mutation observed in DIT1, an enzyme catalyzing the conversion of L-tyrosine to LL-dityrosine for spore wall formation, ${ }^{41}$ may be related to regulation of the shikimate pathway. These mutations, as well as the beneficial effect of restoring the uracil metabolism, outline promising new directions for further rational strain engineering for CCM production, which could be aided by multi-omics analysis, ${ }^{42,43}$ providing more information to improve the prediction and identification of favorable targets for iterative engineering.

The CCM toxicity in S. cerevisiae was characterized as both dosage- and form-dependent. CCM toxicity was clearly more pronounced when the extracellular $\mathrm{pH}$ was near the strongest acidic $\mathrm{pK}_{\mathrm{a}}$ of CCM, 3.64, where the molecule is in a protonated form. This highlighted the necessity of performing fermentations at a constantly optimal $\mathrm{pH}$ condition, for instance $\mathrm{pH}$ 6.0, where CCM is deprotonated. Moreover, even though $S$. cerevisiae cells can tolerate up to $50 \mathrm{~g} / \mathrm{L} \mathrm{CCM}$ in the feeding test, the lethal dosage in the producer strain may be much lower considering that the intracellular CCM concentration may be higher when the strain continuously produces CCM. Adaptive laboratory evolution ${ }^{44}$ or improved CCM export ${ }^{45}$ may be implemented for the isolation of strains that can tolerate $>50 \mathrm{~g} / \mathrm{L} \mathrm{CCM}$, which would be required for a commercial process.

The rational engineering of the Mut131 strain resulted in a strain that could produce $20.8 \mathrm{~g} / \mathrm{L} \mathrm{CCM}$ in a fed-batch fermentation (Figure 5). The improvement through the fermentation process optimization was not only on the CCM titer $(1.59 \mathrm{~g} / \mathrm{L}$ to $5.22-20.8 \mathrm{~g} / \mathrm{L}$ ), but also on the yield (less than 35 to $39.4-70.1 \mathrm{mg} / \mathrm{g}$ glucose) and productivity ( $<21$ to 69.8-139 $\mathrm{mg} / \mathrm{L} / \mathrm{h})$. The strain's performance was largely affected by the medium composition (Figure 5, Figures S9S11) and/or the glucose feeding rate (Figures 5 and 6 and Figure S9), highlighting the potential of lowering the glucose feed rate in improving the production of aromatic compounds.

The CCM/PCA ratio after $72 \mathrm{~h}$ of fermentation was lower in bioreactors (2.1 and 1.5, Figure S9a,b) than in deep-well plates (4.1, Figure 4a). This discrepancy may be related to the different cultivation conditions ${ }^{46}$ and/or medium composition, and the residual PCA could be largely converted to CCM by performing a longer cultivation time (Figures 5c and 6), and complete conversion may likely be realized via further strain engineering (for instance retaining the PCA in the cell) and fermentation optimization.

In summary, we constructed a $S$. cerevisiae strain with the highest CCM titer reported to date through biosensor-aided mutant selection and subsequent rational metabolic engineering. The identified causal mutations expand our understanding of the cellular machinery involved in CCM production. The characterization on these mutations, the associated uracil metabolism, CCM toxicity, and fermentation optimization suggest directions for the further process improvement.

\section{MATERIAL AND METHODS}

Strain Construction. Escherichia coli DH5 $\alpha$ was used as a host for plasmid construction and propagation. Yeast strains (Table S3) derived from CEN.PK $113-7 D^{47}$ were used throughout this study. The yeast strain construction was performed with either marker based selection ${ }^{48}$ or a Cas9assisted approach. ${ }^{49}$ Plasmids (Table S4) were constructed using the EasyClone method ${ }^{48}$ or Gibson assembly with Gibson Assembly Master Mix (New England BioLabs). The plasmids containing both guide RNA cassettes and the donor fragments (Table S5) for mutation introduction were used to construct the strains with point mutations for reverse engineering. DNA fragments for genome integration or gene deletion were constructed by overlap $\mathrm{PCR}^{50}$ (Table S6) or by restriction digestion of EasyClone plasmids.

Yeast transformation was performed using the standard lithium acetate method. ${ }^{51}$ For gene knockout, mutation and genome integration, transformants were subjected to two hour outgrowth on synthetic defined (SD) complete medium before plating onto selective plates. Correct transformants were identified by either genomic PCR validation (knockout and integration) or sequencing of the genomic PCR product (mutation).

Medium and Strain Cultivation. E. coli strains were grown at $37{ }^{\circ} \mathrm{C}$ on Luria-Bertani (LB) medium containing $100 \mathrm{mg} / \mathrm{L}$ ampicillin. Yeast strains were maintained on YPD (10 g/L yeast extract, $20 \mathrm{~g} / \mathrm{L}$ peptone, $20 \mathrm{~g} / \mathrm{L}$ glucose). For strain construction, yeast transformants were selected on either synthetic medium with acetamide as nitrogen source (SM$\left.\mathrm{Ac}^{52}\right)$, YPD with $100 \mathrm{mg} / \mathrm{L}$ nourseothricin, or SD-Ura $(20 \mathrm{~g} / \mathrm{L}$ glucose, $6.7 \mathrm{~g} / \mathrm{L}$ yeast nitrogen base, complete supplement mixture lacking uracil) plates.

Liquid medium was used for yeast cell cultivation for product quantification and strain characterization, including YPD, mineral/DELFT medium ${ }^{48}$ ( $\mathrm{pH}$ 6.0) without or with 20 $\mathrm{mg} / \mathrm{L}$ uracil supplementation, SD-Ura, and FIT medium (mineral medium where $20 \mathrm{~g} / \mathrm{L}$ glucose is substituted with 60 g/L Enpresso EnPump 200 substrate and 0.3\% (v/v) Enpresso Reagent A) without or with $20 \mathrm{mg} / \mathrm{L}$ uracil supplementation.

Yeast strains were cultured at $30{ }^{\circ} \mathrm{C}$ throughout this study, in a 96-deep-well plate at $300 \mathrm{rpm}$ in a New Brunswick Innova 44 shaker or a 24-well plate at $250 \mathrm{rpm}$ in Growth Profiler 960 (EnzyScreen). An overnight preculture of fresh single colonies was subinoculated into $600 \mu \mathrm{L}$ (96-deep well plate) or $1 \mathrm{~mL}$ (24-well plate) medium for subcultivation.

Optical Density and Fluorescence Measurements. Optical density at $600 \mathrm{~nm}$ and fluorescence $\left(\lambda_{\text {excitation }} 485 \mathrm{~nm}\right.$, $\lambda_{\text {emission }} 515 \mathrm{~nm}$ ) of the biosensor output, was measured with a $200 \mu \mathrm{L}$ yeast culture with an appropriate $(2-10 \times)$ dilution using a microtiter plate reader BioTek Synergy MX (BioTek), with the diluted medium as a blank. Since the readouts for cell culture of ST8420 and ST8427 in YPD medium were negative, the data related (Figure 1b) was further processed by calibration with the value for ST8420. Data shown for fluorescence were specific fluorescence, which was normalized by dividing the measured fluorescence value by the measured $\mathrm{OD}_{600}$.

UV Treatment for Mutagenesis Library Generation. A fresh ST8424 colony was inoculated into $2.5 \mathrm{~mL}$ of YPD 
medium in $14 \mathrm{~mL}$ cultivation tube and cultured overnight at $250 \mathrm{rpm}$ at $30^{\circ} \mathrm{C}$. The overnight culture was then washed with sterile water, and the suspension was plated onto a YPD plate for UV treatment. The treatment was performed with a benchtop UV transilluminator (UVP Inc., USA) with $8 \mathrm{~W}$ of light at $302 \mathrm{~nm}$ for $10-20 \mathrm{~s}$. Plates were then placed at $30^{\circ} \mathrm{C}$ under darkness for 2 days before being subject to FACS.

Flow Cytometric Analysis and FACS. Single-cell fluorescence analysis was performed with flow cytometry using the MACSQuant VYB (Miltenyi), on $24 \mathrm{~h}$ subcultures diluted 10-times with water in 96-well microtiter plate. GFP fluorescence from the biosensor was measured after excitation by $488 \mathrm{~nm}$ laser and detected in the $525 / 50 \mathrm{~nm}$ channel. Data on 30000 cells were collected for each sample. Data processing was performed using the FlowJo software.

After two-day growth at $30{ }^{\circ} \mathrm{C}$, UV mutant colonies on the YPD plates were collected by scraping and resuspension in fresh YPD medium. The cell suspension was then inoculated into YPD medium at an initial $\mathrm{OD}_{600}$ of 0.05 , and cultured for $24 \mathrm{~h}$. The $24 \mathrm{~h}$ culture was analyzed and sorted for the first round screening using the BD Biosciences Aria II (Becton Dickinson) with a blue laser $(488 \mathrm{~nm})$. The data for 10000 cells was recorded for mutant and control samples to set up gates for single cells (forward cell scatter, FSC) with maximum and minimum fluorescence output (FITC response). A sampling of 40000 cells were sorted for each group and cultured on YPD medium for $24 \mathrm{~h}$ for a second round of screening following the procedures described above. The sorted cells from the second round were then plated onto a YPD plate to isolate single colonies for subsequent evaluation.

Genome Resequencing and Data Processing. Cells of ST8424 and Mut131 for genome extraction were inoculated from glycerol stock and cultured overnight in $2.5 \mathrm{~mL}$ of YPD in a $14 \mathrm{~mL}$ culture tube. Genome extraction was performed with Quick-DNA Fungal/Bacterial Kit (Zymo research), and the resulting genome samples were further purified with Agencourt AMpure XP magnetic beads (Beckman Coulter). The purified genome samples were subjected to sequencing on NextSeq 500 (Illumina), with NextSeq Mid Output v2 Kit (300 Cycles) (Illumina). Reads of 150 base pair paired-end ( 2 $\times 150 \mathrm{bp}$ ) were collected for a 100 -fold coverage of the $S$. cerevisiae genome size $(12 \mathrm{Mb})$. The raw sequencing data was deposited in the European Nucleotide Archive (accession no. PRJEB32987).

The genome sequence in fastq format was then analyzed with Breseq 0.33.2 $2^{53}$ and Bowtie2 version 2.3.4.3 $3^{54}$ to identify mutations in the mutant strain. The genome of $S$. cerevisiae CEN.PK 113-7D was used as a reference for analysis, and the genome of ST8424 was used as the control to remove falsepositives from the mutant Mut131. In this step, the mutations in the control strain ST8424 were dispatched into the reference genome, and used for the following analysis of Mut131. Furthermore, the predicted mutations in both ST8424 (output to its dispatched genome) and Mut131 were considered as false positives and therefore discarded. Large-scale chromosome duplication or deletion were evaluated by analyzing the coverage maps generated by breseq for each chromosome. To validate the candidate mutations, BLASTn was performed on the mutated genes/intergenic region with reads containing the detected mutations. Only mutations occurring in coding sequences were considered for the reverse engineering. The duplication of heterologous genes for strain engineering was evaluated by checking the read coverage of these genes using breseq. This special breseq analysis was performed on both genome sequence of ST8424 and Mut131. The artificial reference sequence used contains all heterologous genes in ST8424 as well as native S. cerevisiae TKL1 which serves as an indicator for two-copy genes.

Metabolite Quantification. For the quantification of CCM and PCA production, $72 \mathrm{~h}$ subcultures were diluted 5- or 10-times with water and centrifuged at $4000 \mathrm{rpm}$ for $5 \mathrm{~min}$. The supernatant was then analyzed using HPLC with Aminex $\mathrm{HPX}-87 \mathrm{H}$ ion exclusion column kept at $60{ }^{\circ} \mathrm{C}$. The analysis was run with $20 \mu \mathrm{L}$ samples with eluent $1 \mathrm{mM} \mathrm{H}_{2} \mathrm{SO}_{4}$ at a flow of $0.6 \mathrm{~mL} / \mathrm{min}$ for $45 \mathrm{~min}$. The UV detector (Dionex) was used for detection of CCM and PCA at 250 and $220 \mathrm{~nm}$, respectively. The concentrations were quantified by comparison with reference standards.

Fed-Batch Fermentation. Controlled fed-batch fermentations were carried out in $2 \mathrm{~L}$ controlled bioreactors (BioFlo115 from New Brunswick Scientific/Eppendorf) at a controlled temperature of $30{ }^{\circ} \mathrm{C}$ using an electrical heat blanket and an internal cooling coil. The medium for the starting the batch fermentation stage (1.3 L including the inoculum) (BD1 and $\mathrm{BD} 2$ ) contained $10 \mathrm{~g} / \mathrm{L}$ glucose, $10 \mathrm{~g} / \mathrm{L}$ yeast extract, $10 \mathrm{~g} / \mathrm{L}$ $\left(\mathrm{NH}_{4}\right)_{2} \mathrm{SO}_{4}, 4 \mathrm{~g} / \mathrm{L} \mathrm{KH}_{2} \mathrm{PO}_{4}, 0.2 \mathrm{~g} / \mathrm{L} \mathrm{NaCl}, 1.5 \mathrm{~g} / \mathrm{L} \mathrm{MgSO} \mathrm{M}_{4}$. $7 \mathrm{H}_{2} \mathrm{O}, 0.265 \mathrm{~g} / \mathrm{L} \mathrm{CaCl}{ }_{2} \cdot 2 \mathrm{H}_{2} \mathrm{O}$ and trace metal $(10 \mathrm{mg} / \mathrm{L}$ $\mathrm{Na}_{2}$ EDTA, $2 \mathrm{mg} / \mathrm{L} \mathrm{ZnSO}_{4} \cdot 7 \mathrm{H}_{2} \mathrm{O}, 1 \mathrm{mg} / \mathrm{L} \mathrm{CaCl}_{2} \cdot 2 \mathrm{H}_{2} \mathrm{O}, 5 \mathrm{mg} /$ $\mathrm{L} \mathrm{FeSO}_{4} \cdot 7 \mathrm{H}_{2} \mathrm{O}, 0.2 \mathrm{mg} / \mathrm{L} \mathrm{Na}_{2} \mathrm{MoO}_{4} \cdot 2 \mathrm{H}_{2} \mathrm{O}, 0.2 \mathrm{mg} / \mathrm{L} \mathrm{CuSO}$. $5 \mathrm{H}_{2} \mathrm{O}, 0.7 \mathrm{mg} / \mathrm{L} \mathrm{Co}\left(\mathrm{NO}_{3}\right)_{2} \cdot 6 \mathrm{H}_{2} \mathrm{O}, 1.3 \mathrm{mg} / \mathrm{L} \mathrm{MnSO}_{4} \cdot \mathrm{H}_{2} \mathrm{O}$ and $91 \mathrm{mg} / \mathrm{L} \mathrm{MgSO}_{4} \cdot 7 \mathrm{H}_{2} \mathrm{O}$ ). For BD3, BD4, BD5, and BD6, the above medium without glucose was used. An overnight culture of ST8943 in $125 \mathrm{~mL}$ of YPD medium in a $500 \mathrm{~mL}$ shake flask was used for the inoculation. Six different feed compositions were used respectively, (i) solution of $800 \mathrm{~g} / \mathrm{L}$ glucose (BD1), (ii) feed solution containing $800 \mathrm{~g} / \mathrm{L}$ glucose, and $4 \mathrm{~g} / \mathrm{L}$ of $\mathrm{KH}_{2} \mathrm{PO}_{4}$, (iii) feed solution containing $200 \mathrm{~g} / \mathrm{L}$ glucose, $15 \mathrm{~g} / \mathrm{L} \mathrm{KH}_{2} \mathrm{PO}_{4}$ and $8 \mathrm{~g} / \mathrm{L} \mathrm{MgSO}_{4}$ (BD3), (iv) feed solution consisting of $200 \mathrm{~g} / \mathrm{L}$ glucose, $15 \mathrm{~g} / \mathrm{L} \mathrm{KH}_{2} \mathrm{PO}_{4}, 8 \mathrm{~g} / \mathrm{L}$ $\mathrm{MgSO}_{4}, 10 \mathrm{~g} / \mathrm{L}\left(\mathrm{NH}_{4}\right)_{2} \mathrm{SO}_{4}, 20 \mathrm{~g} / \mathrm{L}$ yeast extract, $0.4 \mathrm{~g} / \mathrm{L}$ $\mathrm{CaCl}_{2}$ and $5 \times$ trace metal (BD4), (v) feed solution containing $200 \mathrm{~g} / \mathrm{L}$ glucose, $15 \mathrm{~g} / \mathrm{L} \mathrm{KH}_{2} \mathrm{PO}_{4}, 8 \mathrm{~g} / \mathrm{L} \mathrm{MgSO}_{4}, 10 \mathrm{~g} / \mathrm{L}$ $\left(\mathrm{NH}_{4}\right)_{2} \mathrm{SO}_{4}, 0.4 \mathrm{~g} / \mathrm{L} \mathrm{CaCl}_{2}$ and $5 \times$ trace metal (BD5), or (vi) $1 \times$ feed solution ( $0-48 \mathrm{~h}$ of BD6) with the same composition as $\mathrm{BD} 5$, and $3 \times$ concentrated feed solution $(48-151 \mathrm{~h}$ of $\mathrm{BD} 6)$. In the BD3, BD4, and BD5 runs, $600 \mathrm{~mL}$ of broth was removed from the bioreactor at $56 \mathrm{~h}$, and $250 \mathrm{~mL}$ of broth was additionally removed at $74.2 \mathrm{~h}$ from fermentation BD5. In the BD6 run, $300 \mathrm{~mL}$ of broth was individually removed from the bioreactor at $79.5,103.5,123.5$, and 143.5 h. Filter-sterilized air was fed at $1 \mathrm{vvm}$ through an air sparger. The dissolved oxygen was measured using a polarographic electrode and controlled automatically at $20 \%$ saturation by adjusting the stirring speed. The bioreactors were equipped with two 6-blade Rushton turbines and four baffles. The $\mathrm{pH}$ was controlled at $6.0 \pm 0.1$ by the automatic addition of $\mathrm{H}_{2} \mathrm{SO}_{4} 2 \mathrm{~N}$ or $\mathrm{NH}_{4} \mathrm{OH}$ $14 \%$. A foam sensor was installed, and antifoam (Simethicone $30 \%$, Dow Corning) was added automatically whenever foam was detected. An external pump (Multiflow from Lambda Instruments) was used to add the feed solution. On-line data acquisition and control was performed through dedicated software (Biocommand from New Brunswick Scientific/ Eppendorf) which was interfaced to the feeding pump.

The dry cell weight of all samples was determined by drying a known volume of culture in a weighed dry microtube at 70

${ }^{\circ} \mathrm{C}$ for at least $48 \mathrm{~h}$, after a two-time washing with water. 
Metabolites were quantified by HPLC in sample supernatants, using a Shimadzu LC-20AD instrument, equipped with a refractive index and UV $(210 \mathrm{~nm})$ detectors, serially connected. The analysis was performed on a $20 \mu \mathrm{L}$ sample with eluent $5 \mathrm{mM} \mathrm{H}_{2} \mathrm{SO}_{4}$ at a flow of $0.6 \mathrm{~mL} / \mathrm{min}$, using a Shodex SH1011 column $(8.0 \times 300 \mathrm{~mm})$ kept at $50{ }^{\circ} \mathrm{C}$.

\section{ASSOCIATED CONTENT}

\section{SI Supporting Information}

The Supporting Information is available free of charge at https://pubs.acs.org/doi/10.1021/acssynbio.9b00477.

Supplementary data; information on materials used in this study; introduction of materials and methods associated with acquiring the supplementary data (PDF)

\section{AUTHOR INFORMATION}

\section{Corresponding Author}

Irina Borodina - The Novo Nordisk Foundation Center for Biosustainability, Technical University of Denmark, Lyngby DK-2800 Kgs, Denmark; Email: irbo@biosustain.dtu.dk

\section{Authors}

Guokun Wang - The Novo Nordisk Foundation Center for Biosustainability, Technical University of Denmark, Lyngby DK-2800 Kgs, Denmark; @ orcid.org/0000-0002-6151-3980

Süleyman Øzmerih - The Novo Nordisk Foundation Center for Biosustainability, Technical University of Denmark, Lyngby DK-2800 Kgs, Denmark

Rogério Guerreiro - Biotrend-Inovação e Engenharia em Biotecnologia SA, Cantanhede 3060-197, Portugal

Ana C. Meireles - Biotrend-Inovação e Engenharia em Biotecnologia SA, Cantanhede 3060-197, Portugal

Ana Carolas - Biotrend-Inovação e Engenharia em Biotecnologia SA, Cantanhede 3060-197, Portugal

Nicholas Milne - The Novo Nordisk Foundation Center for Biosustainability, Technical University of Denmark, Lyngby DK-2800 Kgs, Denmark

Michael K. Jensen - The Novo Nordisk Foundation Center for Biosustainability, Technical University of Denmark, Lyngby DK-2800 Kgs, Denmark; @ orcid.org/0000-0001-7574-4707

Bruno S. Ferreira - Biotrend-Inovação e Engenharia em Biotecnologia SA, Cantanhede 3060-197, Portugal; () orcid.org/0000-0002-8565-3101

Complete contact information is available at: https://pubs.acs.org/10.1021/acssynbio.9b00477

\section{Author Contributions}

G.W. and I.B. conceived and designed the study. B.F. and N.M. participated in the design of the work. G.W., S.Ø., R.G., A.C.M., A.C. and N.M. performed the experiments. G.W., S.Ø., M.K.J., B.F., and I.B. analyzed the data. G.W. and I.B. wrote the manuscript and all authors read, edited, and approved the final manuscript.

Notes

The authors declare no competing financial interest.

\section{ACKNOWLEDGMENTS}

This project has received funding from the European Union's Horizon 2020 research and innovation programme under Grant Agreement No. 720770 (DAFIA project). IB also acknowledges the financial support from the Novo Nordisk
Foundation (Grant Agreement No. NNF10CC1016517) and from the European Research Council under the European Union's Horizon 2020 research and innovation programme (YEAST-TRANS project, Grant Agreement No. 757384). We thank Kanchana R. Kildegaard, Mette Kristensen, and Marina Pires for their assistance with HPLC analytics; Mari Cristina Rodriguez de Evgrafov and Jonathan Dahlin for their assistance with flow cytometric analysis; Alexandra Hoffmeyer for her assistance with genome resequencing; Rui Pereira and Emre Özdemir for their assistance with the sequencing data analysis; Robert Mans for his assistance with the growth profiler operation.

\section{REFERENCES}

(1) Rorrer, N. A., Dorgan, J. R., Vardon, D. R., Martinez, C. R., Yang, Y., and Beckham, G. T. (2016) Renewable unsaturated polyesters from muconic acid. ACS Sustainable Chem. Eng. 4, 6867-6876.

(2) Xie, N. Z., Liang, H., Huang, R. B., and Xu, P. (2014) Biotechnological production of muconic acid: current status and future prospects. Biotechnol. Adv. 32, 615-622.

(3) van Duuren, J. B., Wijte, D., Leprince, A., Karge, B., Puchalka, J., Wery, J., Dos Santos, V. A., Eggink, G., and Mars, A. E. (2011) Generation of a catR deficient mutant of $P$. putida KT2440 that produces cis, cis-muconate from benzoate at high rate and yield. $J$. Biotechnol. 156, 163-172.

(4) Kohlstedt, M., Starck, S., Barton, N., Stolzenberger, J., Selzer, M., Mehlmann, K., Schneider, R., Pleissner, D., Rinkel, J., Dickschat, J. S., Venus, J., J, B. J. H. v. D., and Wittmann, C. (2018) From lignin to nylon: Cascaded chemical and biochemical conversion using metabolically engineered Pseudomonas putida. Metab. Eng. 47, 279293.

(5) Vardon, D. R., Franden, M. A., Johnson, C. W., Karp, E. M., Guarnieri, M. T., Linger, J. G., Salm, M. J., Strathmann, T. J., and Beckham, G. T. (2015) Adipic acid production from lignin. Energy Environ. Sci. 8, 617-628.

(6) Kaneko, A., Ishii, Y., and Kirimura, K. (2011) High-yield production of cis,cis-muconic acid from catechol in aqueous solution by biocatalyst. Chem. Lett. 40, 381-383.

(7) Zhang, H., Li, Z., Pereira, B., and Stephanopoulos, G. (2015) Engineering E. coli-E. coli cocultures for production of muconic acid from glycerol. Microb. Cell Fact. 14, 134.

(8) Sun, X., Lin, Y., Huang, Q., Yuan, Q., and Yan, Y. (2013) A novel muconic acid biosynthesis approach by shunting tryptophan biosynthesis via anthranilate. Appl. Environ. Microbiol. 79, 4024-4030.

(9) Weber, C., Bruckner, C., Weinreb, S., Lehr, C., Essl, C., and Boles, E. (2012) Biosynthesis of cis,cis-muconic acid and its aromatic precursors, catechol and protocatechuic acid, from renewable feedstocks by Saccharomyces cerevisiae. Appl. Environ. Microbiol. 78, $8421-8430$.

(10) Zhang, H., Pereira, B., Li, Z., and Stephanopoulos, G. (2015) Engineering Escherichia coli coculture systems for the production of biochemical products. Proc. Natl. Acad. Sci. U. S. A. 112, 8266-8271.

(11) Niu, W., Draths, K. M., and Frost, J. W. (2002) Benzene-free synthesis of adipic acid. Biotechnol. Prog. 18, 201-211.

(12) Fujiwara, R., Noda, S., Tanaka, T., and Kondo, A. (2018) Muconic acid production using gene-level fusion proteins in Escherichia coli. ACS Synth. Biol. 7, 2698-2705.

(13) Wang, S., Bilal, M., Zong, Y., Hu, H. B., Wang, W., and Zhang, X. H. (2018) Development of a plasmid-free biosynthetic pathway for enhanced muconic acid production in Pseudomonas chlororaphis HT66. ACS Synth. Biol. 7, 1131-1142.

(14) Leavitt, J. M., Wagner, J. M., Tu, C. C., Tong, A., Liu, Y., and Alper, H. S. (2017) Biosensor-enabled directed evolution to improve muconic acid production in Saccharomyces cerevisiae. Biotechnol. J. 12, 1600687.

(15) Pyne, M. E., Narcross, L., Melgar, M., Kevvai, K., Mookerjee, S., Leite, G. B., and Martin, V. J. J. (2018) An engineered Arol protein 
degradation approach for increased cis,cis-muconic acid biosynthesis in Saccharomyces cerevisiae. Appl. Environ. Microbiol. 17, e01095-18.

(16) Thompson, B., Pugh, S., Machas, M., and Nielsen, D. R. (2018) Muconic Acid Production via Alternative Pathways and a Synthetic "Metabolic Funnel". ACS Synth. Biol. 7, 565-575.

(17) Sengupta, S., Jonnalagadda, S., Goonewardena, L., and Juturu, V. (2015) Metabolic engineering of a novel muconic acid biosynthesis pathway via 4-hydroxybenzoic acid in Escherichia coli. Appl. Environ. Microbiol. 81, 8037-8043.

(18) Huccetogullari, D., Luo, Z. W., and Lee, S. Y. (2019) Metabolic engineering of microorganisms for production of aromatic compounds. Microb. Cell Fact. 18, 41.

(19) Bui, V., Lau, M. K., MacRae, D., and Schweitzer, D. (2014) Methods for producing isomers of muconic acid and muconate salts. US $8,809,583$.

(20) Skjoedt, M. L., Snoek, T., Kildegaard, K. R., Arsovska, D., Eichenberger, M., Goedecke, T. J., Rajkumar, A. S., Zhang, J., Kristensen, M., Lehka, B. J., Siedler, S., Borodina, I., Jensen, M. K., and Keasling, J. D. (2016) Engineering prokaryotic transcriptional activators as metabolite biosensors in yeast. Nat. Chem. Biol. 12, 951958.

(21) Sonoki, T., Morooka, M., Sakamoto, K., Otsuka, Y., Nakamura, M., Jellison, J., and Goodell, B. (2014) Enhancement of protocatechuate decarboxylase activity for the effective production of muconate from lignin-related aromatic compounds. J. Biotechnol. 192, 71-77.

(22) Weber, H. E., Gottardi, M., Bruckner, C., Oreb, M., Boles, E., and Tripp, J. (2017) Requirement of a functional flavin mononucleotide prenyltransferase for the activity of a bacterial decarboxylase in a heterologous muconic acid pathway in Saccharomyces cerevisiae. Appl. Environ. Microbiol. 83, e03472-16.

(23) Curran, K. A., Leavitt, J. M., Karim, A. S., and Alper, H. S. (2013) Metabolic engineering of muconic acid production in Saccharomyces cerevisiae. Metab. Eng. 15, 55-66.

(24) Suastegui, M., Yu Ng, C., Chowdhury, A., Sun, W., Cao, M., House, E., Maranas, C. D., and Shao, Z. (2017) Multilevel engineering of the upstream module of aromatic amino acid biosynthesis in Saccharomyces cerevisiae for high production of polymer and drug precursors. Metab. Eng. 42, 134-144.

(25) Bruckner, C., Oreb, M., Kunze, G., Boles, E., and Tripp, J. (2018) An expanded enzyme toolbox for production of cis, cismuconic acid and other shikimate pathway derivatives in Saccharomyces cerevisiae. FEMS Yeast Res. 18, No. foy017, DOI: 10.1093/ femsyr/foy017.

(26) Lim, H. G., Jang, S., Jang, S., Seo, S. W., and Jung, G. Y. (2018) Design and optimization of genetically encoded biosensors for highthroughput screening of chemicals. Curr. Opin. Biotechnol. 54, 18-25.

(27) Johnson, A. O., Gonzalez-Villanueva, M., Wong, L., Steinbuchel, A., Tee, K. L., Xu, P., and Wong, T. S. (2017) Design and application of genetically-encoded malonyl-CoA biosensors for metabolic engineering of microbial cell factories. Metab. Eng. 44, 253264.

(28) Li, S., Si, T., Wang, M., and Zhao, H. (2015) Development of a synthetic malonyl-CoA sensor in Saccharomyces cerevisiae for intracellular metabolite monitoring and genetic screening. ACS Synth. Biol. 4, 1308-1315.

(29) Wang, X. N., Cabales, A., Li, Z. H., and Zhang, H. R. (2019) Biosensor-assisted high performing cell selection using an E. coli toxin/antitoxin system. Biochem. Eng. J. 144, 110-118.

(30) Xiao, Y., Bowen, C. H., Liu, D., and Zhang, F. Z. (2016) Exploiting nongenetic cell-to-cell variation for enhanced biosynthesis. Nat. Chem. Biol. 12, 339-344.

(31) Snoek, T., Romero-Suarez, D., Zhang, J., Ambri, F., Skjoedt, M. L., Sudarsan, S., Jensen, M. K., and Keasling, J. D. (2018) An orthogonal and $\mathrm{pH}$-tunable sensor-selector for muconic acid biosynthesis in yeast. ACS Synth. Biol. 7, 995-1003.

(32) Snoek, T., Chaberski, E. K., Ambri, F., Kol, S., Bjorn, S. P., Pang, B., Barajas, J. F., Welner, D. H., Jensen, M. K., and Keasling, J.
D. (2020) Evolution-guided engineering of small-molecule biosensors. Nucleic Acids Res. 48, e3.

(33) Dosil, M., and Bustelo, X. R. (2004) Functional characterization of Pwp2, a WD family protein essential for the assembly of the 90 S pre-ribosomal particle. J. Biol. Chem. 279, 37385-37397.

(34) Livengood, A. J., Zaug, A. J., and Cech, T. R. (2002) Essential regions of Saccharomyces cerevisiae telomerase RNA: separate elements for Est1p and Est2p interaction. Mol. Cell. Biol. 22, 2366-2374.

(35) Dunkler, A., Walther, A., Specht, C. A., and Wendland, J. (2005) Candida albicans CHT3 encodes the functional homolog of the Cts1 Chitinase of Saccharomyces cerevisiae. Fungal Genet. Biol. 42, 935-947.

(36) Watts, T., Khalimonchuk, O., Wolf, R. Z., Turk, E. M., Mohr, G., and Winge, D. R. (2011) Mne1 is a novel component of the mitochondrial splicing apparatus responsible for processing of a COX1 group I intron in yeast. J. Biol. Chem. 286, 10137-10146.

(37) Sardi, M., Paithane, V., Place, M., Robinson, E., Hose, J., Wohlbach, D. J., and Gasch, A. P. (2018) Genome-wide association across Saccharomyces cerevisiae strains reveals substantial variation in underlying gene requirements for toxin tolerance. PLoS Genet. 14, e1007217.

(38) Cheong, H., Nair, U., Geng, J. F., and Klionsky, D. J. (2008) The Atg1 kinase complex is involved in the regulation of protein recruitment to initiate sequestering vesicle formation for nonspecific autophagy in Saccharomyces cerevisiae. Mol. Biol. Cell 19, 668-681.

(39) Matsui, A., Kamada, Y., and Matsuura, A. (2013) The role of autophagy in genome stability through suppression of abnormal mitosis under starvation. PLoS Genet. 9, e1003245.

(40) Bardin, A. J., Boselli, M. G., and Amon, A. (2003) Mitotic exit regulation through distinct domains within the protein kinase Cdc15. Mol. Cell. Biol. 23, 5018-5030.

(41) Briza, P., Eckerstorfer, M., and Breitenbach, M. (1994) The sporulation-specific enzymes encoded by the DIT1 and DIT2 genes catalyze a two-step reaction leading to a soluble LL-dityrosinecontaining precursor of the yeast spore wall. Proc. Natl. Acad. Sci. U. S. A. 91, 4524-4528.

(42) Lee, J. W., Na, D., Park, J. M., Lee, J., Choi, S., and Lee, S. Y. (2012) Systems metabolic engineering of microorganisms for natural and non-natural chemicals. Nat. Chem. Biol. 8, 536-546.

(43) Rodriguez, A., Chen, Y., Khoomrung, S., Ozdemir, E., Borodina, I., and Nielsen, J. (2017) Comparison of the metabolic response to over-production of $p$-coumaric acid in two yeast strains. Metab. Eng. 44, 265-272.

(44) Pereira, R., Wei, Y., Mohamed, E., Radi, M., Malina, C., Herrgard, M. J., Feist, A. M., Nielsen, J., and Chen, Y. (2019) Adaptive laboratory evolution of tolerance to dicarboxylic acids in Saccharomyces cerevisiae. Metab. Eng. 56, 130-141.

(45) Borodina, I. (2019) Understanding metabolite transport gives an upper hand in strain development. Microb. Biotechnol. 12, 69-70.

(46) Wehrs, M., Tanjore, D., Eng, T., Lievense, J., Pray, T. R., and Mukhopadhyay, A. (2019) Engineering robust production microbes for large-scale cultivation. Trends Microbiol. 27, 524-537.

(47) Entian, K. D., and Kotter, P. (2007) Yeast genetic strain and plasmid collections. Methods Microbiol. 36, 629-666.

(48) Jensen, N. B., Strucko, T., Kildegaard, K. R., David, F., Maury, J., Mortensen, U. H., Forster, J., Nielsen, J., and Borodina, I. (2014) EasyClone: method for iterative chromosomal integration of multiple genes in Saccharomyces cerevisiae. FEMS Yeast Res. 14, 238-248.

(49) Jessop-Fabre, M. M., Jakociunas, T., Stovicek, V., Dai, Z., Jensen, M. K., Keasling, J. D., and Borodina, I. (2016) EasyCloneMarkerFree: A vector toolkit for marker-less integration of genes into Saccharomyces cerevisiae via CRISPR-Cas9. Biotechnol. J. 11, 11101117.

(50) Zhou, Y. J., Gao, W., Rong, Q., Jin, G., Chu, H., Liu, W., Yang, W., Zhu, Z., Li, G., Zhu, G., Huang, L., and Zhao, Z. K. (2012) Modular pathway engineering of diterpenoid synthases and the mevalonic acid pathway for miltiradiene production. J. Am. Chem. Soc. $134,3234-3241$. 
(51) Gietz, R. D., and Woods, R. A. (2002) Transformation of yeast by lithium acetate/single-stranded carrier DNA/polyethylene glycol method. Methods Enzymol. 350, 87-96.

(52) Solis-Escalante, D., Kuijpers, N. G., Bongaerts, N., Bolat, I., Bosman, L., Pronk, J. T., Daran, J. M., and Daran-Lapujade, P. (2013) amdSYM, a new dominant recyclable marker cassette for Saccharomyces cerevisiae. FEMS Yeast Res. 13, 126-139.

(53) Deatherage, D. E., and Barrick, J. E. (2014) Identification of mutations in laboratory-evolved microbes from next-generation sequencing data using breseq. Methods Mol. Biol. 1151, 165-188.

(54) Langmead, B., and Salzberg, S. L. (2012) Fast gapped-read alignment with Bowtie 2. Nat. Methods 9, 357-U354. 